'Омский государственный медицинский университет, Омск, Россия

${ }^{2}$ Корпоративный фонд «University Medical Center», Нур-Султан, Казахстан

${ }^{3}$ Медицинский университет Астана, Нур-Султан, Казахстан

4Лаборатория геномной и персонализированной медицины «Национальная Лаборатория Астана» Назарбаев Университета, Нур-Султан, Казахстан

В последние десятилетия неуклонно растет распространенность ожирения в большинстве стран мира. Избыточная масса тела является фактором риска широкого спектра эндокринных, сердечно-сосудистых, желудочно-кишечных, метаболических, неопластических и мышечно-скелетных нарушений и заболеваний. Как известно, ожирение - это состояние хронического воспаления и сильного окислительного стресса, что непременно оказывает влияние на длину теломер хромосом. Динамика изменений длины теломер играет решающую роль в регуляции клеточных процессов и клеточных изменений. Повреждение теломер, структур хроматина, помогающих поддерживать стабильность генома, приводит к гибели клеток или к их старению. Однако сведения о том, как изменяется длина теломер после снижения массы тела посредством бариатрических операций, остаются до настоящего времени ограниченными. Существует несколько видов бариатрических операций, каждый из которых имеет свои преимущества и недостатки. Исходя из этого, возможно, восстановление длины теломер будет отличаться в зависимости от использованной методики. В данном обзоре литературы описываются механизмы укорочения длины теломер лейкоцитов и то, как на это могут повлиять бариатрические операции. Обзор также включает в себя анализ доказательств, связывающих ожирение и ускоренные процессы старения, поскольку они регулируются теломерами.

КЛЮЧЕВЫЕ СЛОВА: длина теломер; старение; бариатрическая хирургия; ожирение; метаболический синдром.

\title{
THE EVALUATION OF CHROMOSOME TELOMERE LENGTH CHANGE AS A CRITERION OF LIFE EXPECTANCY IN BARIATRIC PRACTICE
}

\author{
(c) Farida K. Bekmurzinova1*, Oral B. Ospanov ${ }^{2,3}$, Ainur R. Akilzhanova4, Ulan A. Kozhamkulov, Saule E. Rakhimova4
}

'Omsk State Medical University, Omsk, Russia

2University Medical Center, Nur-Sultan, Kazakhstan

${ }^{3}$ Astana Medical University, Nur-Sultan, Kazakhstan

${ }^{4}$ Laboratory of Genomic and Personalized Medicine «National Laboratory Astana» Nazarbayev University, Nur-Sultan,

Kazakhstan

In recent decades, the prevalence of obesity has been steadily increasing in most countries of the world. Overweight is a risk factor for a wide range of endocrine, cardiovascular, gastrointestinal, metabolic, neoplastic and musculoskeletal disorders and diseases. As you know, obesity is a state of chronic inflammation and severe oxidative stress, which will certainly affect the length of the chromosome telomeres. The dynamics of telomere length changes plays a decisive role in the regulation of cellular processes and cellular changes. Damage to telomeres, chromatin structures that help maintain the stability of the genome, leads to cell death or aging. However, information on how telomere length changes after weight loss through bariatric surgery remains limited to date. There are several types of bariatric surgery, each of which has its advantages and disadvantages. Based on this, it is possible that the restoration of the telomere length will differ depending on the technique used. This review describes the mechanisms for shortening leukocyte telomeres, and how bariatric surgery can affect this. The review also includes an analysis of evidence linking obesity and accelerated aging processes, as they are regulated by telomeres.

KEYWORDS: telomere length; aging; bariatric surgery; obesity; metabolic syndrome.

\section{ВВЕДЕНИЕ}

Вопрос о продолжительности жизни пациентов с метаболическим синдромом в последние десятилетия является весьма актуальным, поскольку один из главных компонентов данного синдрома, ожирение, значится в списке главных причин преждевременной смертности населения. По данным Всемирной организации здра- воохранения (ВО3), опубликованным в 2016 г., более 1,9 млрд взрослых старше 18 лет имели избыточный вес. Из них свыше 650 млн страдали ожирением. Ежегодно в мире от коморбидных ожирению заболеваний умирают около 2,8 млн человек. Темпы увеличения числа больных, страдающих ожирением, настолько велики, что данное заболевание приобретает характер неинфекционной эпидемии. Ожирение является условием, при 
котором происходит чрезмерное накопление жировой ткани, что может оказывать неблагоприятное воздействие на здоровье и приводить к уменьшению ожидаемой продолжительности жизни. В соответствии с данными ВО3, ожирением обусловлено 44\% случаев развития сахарного диабета 2 типа (СД2), 23\% ишемической болезни сердца (ИБС) и 7-41\% определенных видов рака [1]. Эти расстройства рассматриваются как следствие повышенного окислительного стресса и воспаления в организме.

Увеличение окислительного стресса и воспаления влияет на длину теломер и может ускорить старение, поэтому теломеры являются ключевыми маркерами биологического старения [2]. Они представляют собой специализированные ДНК-белковые структуры, обнаруженные на концах эукариотических хромосом. Длина теломер играет важную роль в регуляции клеточных процессов и клеточной гибели, по состоянию теломер определяют не только продолжительность жизни одной клетки, но и состояние органов, систем и организма в целом.

Целью данного обзора литературы является критическая оценка и обобщение научных знаний о том, как изменяется продолжительность жизни пациентов с метаболическим синдромом после снижения массы тела.

При подготовке литературного обзора нами проведен анализ публикаций за последние 10 лет. Для поиска источников использовали базу данных PubMed, а также сайты издательств Springer и Elsiver для доступа к полнотекстовым версиям статей. Поиск публикаций осуществляли по соответствующим ключевым словам. Для PubMed: telomere length; aging; bariatric surgery; obesity; metabolic syndrome.

\section{ГОМЕОСТАЗ ДЛИНЫ ТЕЛОМЕР}

Теломеры представляют собой структуры ДНК, состоящие из тандемных повторов последовательности TTAGGG и ассоциированных белков на концах хромосом человека. ДНК теломер поддерживается высокорегулируемым действием клеточного фермента теломеразы. Посредством этого механизма хромосомная ДНК и гены защищены от повреждений во время процесса деления клетки [3]. Фермент теломераза - это специфический рибонуклеопротеин клетки, который в конечном счете работает как обратная транскриптаза и принимает участие в синтезе активных центров для связывания теломерной ДНК с теломерными защитными белками.

Удлинение теломер происходит в эмбриональном периоде, когда активен фермент теломераза и далее остается активным постнатально в клетках зародышевого пути и в некоторых стволовых клетках [4]. Этот процесс может быть активирован в тканях, которые требуют дальнейшего расширения количества клеток, например, в гемопоэтической системе [4]. В исследовании Gadalla et al. [5] наблюдается увеличение выживаемости реципиентов с трансплантацией костного мозга по поводу лечения апластической анемии, когда донорские ткани обладали более длинными теломерами. Это увеличение выживаемости связано с усилением теломерной способности, которая помогла восстановить гемопоэтическую систему.

Как известно, в S-фазу клеточного цикла происходит репликация ДНК, в результате чего каждый раз проис- ходит недорепликация короткого концевого участка хроматиды. Для того чтобы каждый период репликации не сопровождался потерей значимых последовательностей ДНК, на концах хромосом возникли незначимые последовательности ДНК - теломеры [6]. Укорочение теломер - это механизм, который происходит вследствие нормального деления клеток и репликационного механизма. Данный механизм демонстрируется на опухолевых клетках [7], которые были модифицированы имели длинные теломеры и высокую активность теломеразы; в лейкоцитах, которые были стимулированы митогеном [8], и в стволовых клетках человека [9]. Таким образом, в норме фермент теломераза активен только в эмбриональном периоде, далее в постэмбриональном периоде происходит только сокращение длины теломер в процессе репликации.

\section{МЕХАНИЗМЫ УКОРОЧЕНИЯ ТЕЛОМЕР}

Теломерная ДНК чувствительна к естественной терминальной эрозии за счет различных процессов, включая механизм репликации линейной хромосомной ДНК, которая приводит к уменьшению длины теломер с каждым делением клеток [10], а также процессов, связанных с действием нуклеазы, репликации, рекомбинации ДНК и действием окислительного стресса. Несмотря на то что фермент теломераза способен противодействовать укорочению длины теломер, активность его обычно отсутствует в нормальных клетках взрослого человека. Когда длина теломер уменьшается до критического значения, это приводит к репликативному старению и гибели клеток $[11,12]$. Генетическая предрасположенность к более длинным теломерам связана с повышенным риском развития рака [13-15].

В нескольких независимых исследованиях было показано, что уменьшение длины теломерной ДНК человека связано с широким спектром различных заболеваний и для нескольких возрастных групп больных, где можно прогнозировать будущие риски и исходы, включая смертность.

У пожилых людей происходит укорочение теломер во всех клетках крови, но скорость укорочения у всех разная. Изменение длины теломер с возрастом - нестабильный процесс, так, например, наиболее резкое уменьшение длины в лимфоцитах происходит в течение первого года жизни, затем этот процесс замедляется и приблизительно после 30 лет имеет постоянный уровень [16].

При некоторых генетических заболеваниях человека, таких как синдром Дауна, синдром Ди Джорджи, а также при врожденном дискератозе происходит раннее сокращение длины теломер, и это обусловлено несколькими мутациями в гене субъединицы hTERT теломеразы, которая стабилизирует длину теломер $[17,18]$.

Имеются данные, где показана роль воздействия стресса и его влияние на быстрое укорочение теломер в клетках, и этот эффект зависел от продолжительности и интенсивности воздействия стресса [19]. Повреждается хромосома, ДНК теломеры, а также уменьшается длина теломеры при оксидативном стрессе, независимо от причин, вызвавших стресс. Стаж курения напрямую влияет на степень укорочения теломер, так, чем стаж 
курения больше, тем сильнее происходит сокращение длины теломер в лимфоцитах [20].

Можно сказать, что изменение длины теломер связано с развитием сердечно-сосудистых (СС3) и инфекционных заболеваний $[21,22]$. Риск смертности от ССЗ у больных с более короткими теломерами в лейкоцитах крови увеличен в 3 раза, а от инфекционных - в 8 раз [23-25]. Такие же риски смертности отмечены при сахарном диабете 2 типа, что также связано с окислительным стрессом, который, в свою очередь, влияет на длину теломер клеток крови [26].

Бесспорно, ожирение влияет на длину теломер и может ускорить процесс старения. Имеется четкая связь между индексом массы тела (ИМТ) и длиной теломер лейкоцитов, которая варьируется в зависимости от возраста. Ожирение рассматривается как основной детерминант в регуляции старения жировой ткани наряду с метаболическими изменениями, такими как повышение провоспалительных цитокинов, развитие инсулинорезистентности, сахарный диабет 2 типа и СС3. По результатам исследования [27] становится ясно, что ИМТ имеет значительную корреляцию с длиной теломер у пациентов моложе 60 лет.

\section{ОПЕРАТИВНОЕ ЛЕЧЕНИЕ ОЖИРЕНИЯ И ДЛИНА ТЕЛОМЕР}

Распространенность ожирения связана с возрастающей урбанизацией, снижением физической активности и легкой доступностью высококалорийных продуктов. В связи с этим возникает необходимость поиска эффективных способов снижения массы тела.

Традиционно основным методом лечения ожирения был консервативный, однако данный вид терапии носит непродолжительный характер и малоэффективен. С середины XX в. стали разрабатываться хирургические методы коррекции избыточной массы тела. Бариатрическая хирургия сформировалась как отдельная область знаний. Хирургия ожирения широко признана и является наиболее эффективным видом лечения. Это единственный известный метод, который приводит к значительной потере веса и предотвращает его восстановление. В мировой практике используют десятки различных бариатрических операций. Ежегодно проводится более 500000 хирургических вмешательств по поводу снижения избыточной массы тела. Их них продольная резекция желудка составляет 49\%, гастрошунтирование по Ру - 43\% всех выполняемых бариатрических операций [28-30].

Основываясь на исследовании [31], авторы утверждают, что после операции гастрошунтирования по Ру пациенты показали значительное снижение ИМТ, и наблюдалось увеличение длины теломер, а также снижение в них окислительного стресса. Следовательно, данная методика операции улучшает фенотип преждевременного старения.

Caetano de Queiroz и соавт. [32] провели исследование, основанное на наблюдении за 120 пациентами через 2 года после гастрошунтирования по Ру, по результатам которого были доказаны эффективность и безопасность операции в борьбе с ожирением и связанными с ним состояниями. Смертность была равна нулю. Среднее значение потери лишнего веса EWL (Excess Weight Loss) составило 75\%. При этом в 4,2\% случаях имелись осложнения.

\section{ОБСУЖДЕНИЕ}

После хирургического лечения ожирения пациенты резко теряют массу тела в основном в первые 1-2 года [33]. В результате чего происходит снижение уровня провоспалительных цитокинов [34], что влияет В той или иной степени на длину теломер. В исследовании Formichi et al. выявили уменьшение длины через 1 год после хирургического лечения ожирения [35]. Однако Laimer et al., наоборот, отмечали увеличение длины теломер в течение 10 лет после бариатрической операции [36]. Также удлинение длины теломер через 3-5 лет после операции наблюдали другие авторы [37]. Следовательно, нами в результате анализа литературы были получены противоречивые данные по изменению длины теломер.

Вместе с тем важно отметить, что, по данным литературных источников, ключевой особенностью гастрошунтирования является то, что эта операция позволяет пациентам поддерживать значительное снижение массы тела в долгосрочной перспективе. Однако эффекты бариатрической хирургии не ограничиваются только потерей веса, также отмечается улучшение функции жировой ткани и изменение чувствительности к инсулину [38]. По данным ряда авторов [31], выявляется значительное снижение провоспалительных маркеров, таких как интерлейкин-6 и С-реактивный белок у пациентов через 2 года после операции. Это способствует уменьшению окислительного стресса, что предотвращает преждевременное старение и гибель клеток.

Насколько нам известно, в данный момент в мире нет исследований по изучению изменению длины теломер лейкоцитов после бесстеплерных типов гастрошунтирования, которые могут быть наиболее доступными и вместе с тем не менее эффективными, чем бариатрические и метаболические операции по степлерной технологии [39-40]. Но вместе с тем известно, что такие исследования планируется провести в ближайшие годы [41].

Таким образом, результаты представленного литературного обзора указывают на то, что в настоящее время в мире отмечается глобальная тенденция к росту ожирения, ведется множество исследований по лечению и профилактике осложнений данной патологии.

Проанализировав результаты вышеуказанных исследований, стоит сказать о том, что в настоящий момент имеется недостаточное количество знаний в этой области. В доступной литературе было найдено всего 5 публикаций о том, как изменяется длина теломер лейкоцитов, а следовательно, продолжительность жизни после снижения массы тела посредством бариатрических операций, по итогам которых были получены противоречивые данные. Одни авторы утверждают, что длина теломер не увеличивается, а наоборот, уменьшается вследствие катаболического состояния после снижения массы тела [35]. А немало других авторов, которые считают, что происходит значительное восстановление длины теломер, которое связано с уменьшением окислительного процесса в клетках после бариатрической хирургии $[31,36,37]$.

Следовательно, данный аспект диктует необходимость продолжения исследования в этом направлении 
с целью выявления изменения длины хромосомных теломер при ожирении в зависимости от степени снижении массы тела.

\section{ЗАКЛЮЧЕНИЕ}

В ходе обзора литературы была выявлена патогенетическая значимость длины теломер лейкоцитов в продолжительности жизни пациентов с метаболическим синдромом после бариатрических операций. Также было установлено, что после значительного снижения массы тела наблюдается чаще всего удлинение теломер лейкоцитов. Однако требуются дополнительные исследования, которые могли бы установить влияние снижения массы тела при ожирении на продолжительность жизни и, возможно, раскрыть важные механизмы, лежащие в основе долголетия.

\section{ДОПОЛНИТЕЛЬНАЯ ИНФОРМАЦИЯ}

Источник финансирования. Подготовка и публикация рукописи проведены на личные средства авторского коллектива.

Конфликт интересов. Авторы декларируют отсутствие явных и потенциальных конфликтов интересов, связанных с публикацией настоящей статьи.

Участие авторов. Бекмурзинова Ф.К. - сбор, обработка и анализ публикаций, написание основного текста статьи; Оспанов О.Б. - критический пересмотр содержания статьи, написание текста в разделе «Оперативное лечение ожирения и длина теломер», а также раздела «Обсуждение»; Акильжанова А.Р. - редактирование текста статьи в части вопросов, касающихся гомеостаза длины теломер; Кожамкулов У.А. редактирование текста статьи в части механизмов укорочения длины теломер; Рахимова С.Е. - составление резюме публикации. Все авторы внесли значимый вклад в проведение исследования и подготовку статьи, прочли и одобрили финальную версию статьи перед публикацией.

\section{СПИСОК ЛИТЕРАТУРЫ | REFERENCES}

1. Metabolic mediators of the effects of body-mass index, overweight, and obesity on coronary heart disease and stroke: a pooled analysis of 97 prospective cohorts with 1.8 million participants. Lancet. 2014;383(9921):970-983 doi: https://doi.org/10.1016/s0140-6736(13)61836-x

2. Tzanetakou IP, Katsilambros NL, Benetos A, et al. «ls obesity linked to aging?»: adipose tissue and the role of telomeres. Ageing Res Rev. 2012;11(2):220-229. doi: https://doi.org/10.1016/j.arr.2011.12.003

3. Paul L. Diet, nutrition and telomere length. J Nutr Biochem. 2011;22(10):895-901.

doi: https://doi.org/10.1016/j.jnutbio.2010.12.001

4. Reddel RR. Telomere maintenance mechanisms in cancer: clinical implications. Curr Pharm Des. 2014;20(41):6361-6374 doi: https://doi.org/10.2174/1381612820666140630101047

5. Gadalla SM, Wang T, Haagenson M, et al. Association between donor leukocyte telomere length and survival after unrelated allogeneic hematopoietic cell transplantation for severe aplastic anemia. JAMA. 2015;313(6):594-602. doi: https://doi.org/10.1001/jama.2015.7

6. Fasching CL. Telomere length measurement as a clinical biomarker of aging and disease. Crit Rev Clin Lab Sci. 2018;55(7):443-465 doi: https://doi.org/10.1080/10408363.2018.1504274

7. Pickett HA, Cesare AJ, Johnston RL, et al. Control of telomere length by a trimming mechanism that involves generation of t-circles. EMBO J. 2009;28(7):799-809. doi: https://doi.org/10.1038/emboj.2009.42

8. Pickett HA, Henson JD, Au AY, et al. Normal mammalian cells negatively regulate telomere length by telomere trimming. Hum Mol Genet. 2011:20(23):4684-4692. doi: https://doi.org/10.1093/hmg/ddr402

9. Rivera T, Haggblom C, Cosconati S, Karlseder J. A balance between elongation and trimming regulates telomere stability in stem cells. Nat Struct Mol Biol. 2017;24(1):30-39. doi: https://doi.org/10.1038/nsmb.3335

10. Blackburn EH. Telomeres and telomerase: their mechanisms of action and the effects of altering their functions. FEBS Lett. 2005;579(4):859862. doi: https://doi.org/10.1016/j.febslet.2004.11.036

11. Lopez-Otin C, Blasco MA, Partridge L, et al. The hallmarks of aging. Cell. 2013;153(6):1194-1217. doi: https://doi.org/10.1016/j.cell.2013.05.039

12. Armanios M, Blackburn EH. The telomere syndromes. Nat Rev Genet. 2012;13(10):693-704. doi: https://doi.org/10.1038/nrg3246

13. Blackburn EH, Epel ES, Lin J. Human telomere biology: A contributory and interactive factor in aging, disease risks, and protection. Science. 2015;350(6265):1193-1198. doi: https://doi.org/10.1126/science.aab3389

14. Bojesen SE, Pooley KA, Johnatty SE, et al. Multiple independent variants at the TERT locus are associated with telomere length and risks of breast and ovarian cancer. Nat Genet. 2013;45(4):371-384, 384e371-372. doi: https://doi.org/10.1038/ng.2566

15. Walsh KM, Codd V, Smirnov IV, et al. Variants near TERT and TERC influencing telomere length are associated with high-grade glioma risk. Nat Genet. 2014;46(7):731-735 doi: https://doi.org/10.1038/ng.3004
16. Aviv A, Chen W, Gardner JP, et al. Leukocyte telomere dynamics: longitudinal findings among young adults in the Bogalusa Heart Study. Am J Epidemiol. 2009;169(3):323-329. doi: https://doi.org/10.1093/aje/kwn338

17. Nelson ND, Bertuch AA. Dyskeratosis congenita as a disorder of telomere maintenance. Mutat Res. 2012;730(1-2):43-51 doi: https://doi.org/10.1016/j.mrfmmm.2011.06.008

18. Albizua I, Rambo-Martin BL, Allen EG, et al. Association between telomere length and chromosome 21 nondisjunction in the oocyte. Hum Genet. 2015;134(11-12):1263-1270. doi: https://doi.org/10.1007/s00439-015-1603-0

19. Lin J, Epel E, Blackburn E. Telomeres and lifestyle factors: roles in cellular aging. Mutat Res. 2012;730(1-2):85-89. doi: https://doi.org/10.1016/j.mrfmmm.2011.08.003

20. Valdes AM, Andrew T, Gardner JP, et al. Obesity, cigarette smoking, and telomere length in women. Lancet. 2005;366(9486):662-664. doi: https://doi.org/10.1016/s0140-6736(05)66630-5

21. Raschenberger J, Kollerits B, Hammerer-Lercher A, et al. The association of relative telomere length with symptomatic peripheral arterial disease: results from the CAVASIC study. Atherosclerosis. 2013;229(2):469-474. doi: https://doi.org/10.1016/j.atherosclerosis.2013.05.027

22. Mainous AG, 3rd, Codd V, Diaz VA, et al. Leukocyte telomere length and coronary artery calcification. Atherosclerosis. 2010;210(1):262-267. doi: https://doi.org/10.1016/j.atherosclerosis.2009.10.047

23. Cawthon RM, Smith KR, O'Brien E, et al. Association between telomere length in blood and mortality in people aged 60 years or older. Lancet. 2003;361(9355):393-395. doi: https://doi.org/10.1016/s0140-6736(03)12384-7

24. Zanet DL, Thorne A, Singer J, et al. Association Between Short Leukocyte Telomere Length and HIV Infection in a Cohort Study: No Evidence of a Relationship With Antiretroviral Therapy. Clin Infect Dis. 2014;58(9):1322-1332. doi: https://doi.org/10.1093/cid/ciu051

25. van de Berg PJ, Griffiths SJ, Yong SL, et al. Cytomegalovirus infection reduces telomere length of the circulating T cell pool. J Immunol. 2010;184(7):3417-3423. doi: https://doi.org/10.4049/jimmunol.0903442

26. Willeit $P$, Raschenberger J, Heydon EE, et al. Leucocyte telomere length and risk of type 2 diabetes mellitus: new prospective cohort study and literaturebased meta-analysis. PLoS One. 2014;9(11):e112483 doi: https://doi.org/10.1371/journal.pone.0112483

27. Muezzinler A, Mons U, Dieffenbach AK, et al. Body mass index and leukocyte telomere length dynamics among older adults: Results from the ESTHER cohort. Exp Gerontol. 2016;74:1-8. doi: https://doi.org/10.1016/j.exger.2015.11.019

28. Angrisani L, Santonicola A, lovino P, et al. Bariatric Surgery Worldwide 2013. Obes Surg. 2015;25(10):1822-1832. doi: https://doi.org/10.1007/s11695-015-1657-z

29. Khorgami Z, Andalib A, Corcelles R, et al. Recent National Trends In The Surgical Treatment of Obesity: Sleeve Gastrectomy Dominates. Surg Obes Relat Dis. 2015;11(6):S6-S8. doi: https://doi.org/10.1016/j. soard.2015.10.012 
30. Nicoletti C, Cortes-Oliveira C, Pinhel M, Nonino C. Bariatric Surgery and Precision Nutrition. Nutrients. 2017;9(9):974. doi: https://doi.org/10.3390/nu9090974

31. Hohensinner PJ, Kaun C, Ebenbauer B, et al. Reduction of Premature Aging Markers After Gastric Bypass Surgery in Morbidly Obese Patients. Obes Surg. 2018;28(9):2804-2810. doi: https://doi.org/10.1007/s11695-018-3247-3

32. Queiroz C, Sallet JA, PG DEBES, et al. Application of BAROS questionnaire in obese patients undergoing bariatric surgery with 2 years of evolution. Ara Gastroenterol. 2017;54(1):60-64. doi: https://doi.org/10.1590/S0004-2803.2017v54n1-12

33. Sjostrom L. Review of the key results from the Swedish Obese Subjects (SOS) trial - a prospective controlled intervention study of bariatric surgery. J Intern Med. 2013;273(3):219-234. doi: https://doi.org/10.1111/joim.12012

34. Barazzoni R, Palmisano S, Gortan Cappellari G, et al. Gastric bypassinduced weight loss alters obesity-associated patterns of plasma pentraxin-3 and systemic inflammatory markers. Surg Obes Relat Dis. 2016;12(1):23-32. doi: https://doi.org/10.1016/j.soard.2015.04.013

35. Formichi C, Cantara S, Ciuoli C, et al. Weight loss associated with bariatric surgery does not restore short telomere length of severe obese patients after 1 year. Obes Surg. 2014;24(12):2089-2093. doi: https://doi.org/10.1007/s11695-014-1300-4
36. Laimer M, Melmer A, Lamina C, et al. Telomere length increase after weight loss induced by bariatric surgery: results from a 10 year prospective study. Int J Obes (Lond). 2016;40(5):773-778. doi: https://doi.org/10.1038/ijo.2015.238

37. Dershem R, Chu X, Wood GC, et al. Changes in telomere length 3-5 years after gastric bypass surgery. Int J Obes (Lond). 2017;41(11):1718-1720. doi: https://doi.org/10.1038/ijo.2017.156

38. Frikke-Schmidt $\mathrm{H}, \mathrm{O}$ 'Rourke RW, Lumeng $\mathrm{CN}$, et al. Does bariatric surgery improve adipose tissue function? Obes Rev. 2016;17(9):795-809. doi: https://doi.org/10.1111/obr.12429

39. Ospanov O, Buchwald JN, Yeleuov G, Bekmurzinova F. Laparoscopic One-Anastomosis Gastric Bypass with BandSeparated Gastric Pouch (OAGB-BSGP): a Randomized Controlled Trial. Obes Surg. 2019;29(12):4131-4137. doi: https://doi.org/10.1007/s11695-019-04236-1

40. Ospanov OB. Surgical technique of laparoscopic mini-gastric bypass with obstructive stapleless pouch creation: A case series. Int J Surg. 2019;67:70-75. doi: https://doi.org/10.1016/j.jjsu.2019.05.011

41. Ospanov O, Yeleuov G, Kadyrova I, Bekmurzinova F. The life expectancy of patients with metabolic syndrome after weight loss: study protocol for a randomized clinical trial (LIFEXPE-RT). Trials. 2019;20(1):202. doi: https://doi.org/10.1186/s13063-019-3304-9

\section{ИНФОРМАЦИЯ ОБ АВТОРАХ [AUTHORS INFO]:}

*Бекмурзинова Фарида Кайратовна, клинический ординатор [Farida K. Bekmurzinova, resident]; адрес: Россия, 644036, Омск, ул. Мельничная, д. 58B [address: 58V Melnichnaya street, 644036 Omsk, Russia]; ORCID: https://orcid.org/0000-0002-6634-5728; eLilbrary SPIN: 7214-9199; e-mail: farida.bariatric@gmail.com

Оспанов Орал Базарбаевич, д.м.н., профессор [Oral B. Ospanov, MD, PhD, Professor]; ORCID: https://orcid.org/000-0002-1840-114X; eLilbrary SPIN: 9940-6026; e-mail: o_ospanov@mail.ru Акильжанова Айнур Рахметуловна, д.м.н., профессор [Ainur R. Akilzhanova, MD, PhD, Professor]; ORCID: https://orcid.org/0000-0001-6161-8355; eLilbrary SPIN: 8870-6389; e-mail: akilzhanova@nu.edu.kz

Кожамкулов Улан Анетович, к.м.н. [Ulan A. Kozhamkulov, MD, PhD]; ORCID: https://orcid.org/0000-0002-9782-7631; eLilbrary SPIN: 5253-3270; e-mail: ulan.kozhamkulov@nu.edu.kz

Рахимова Сауле Есламовна, к.б.н. [Saule E. Rakhimova, PhD in biology]; ORCID: https://orcid.org/0000-0002-8245-2400; eLilbrary SPIN: 3486-4510; e-mail: saule.rakhimova@nu.edu.kz

*Автор, ответственный за переписку / Corresponding author.

\section{ЦИТИРОВАТЬ:}

Бекмурзинова Ф.К., Оспанов О.Б., Акильжанова А.Р., Кожамкулов У.А., Рахимова С.Е. Оценка изменения длины теломер хромосом как критерий продолжительности жизни в бариатрической практике // Ожирение и метаболизм. - 2020. T. 17. - №2. - C. 125-129. doi: https://doi.org/10.14341/omet10331

\section{TO CITE THIS ARTICLE:}

Bekmurzinova FK, Ospanov OB, Akilzhanova AR, Kozhamkulov UA, Rakhimova SE. The evaluation of chromosome telomere length change as a criterion of life expectancy in bariatric practice. Obesity and metabolism. 2020;17(2):125-129. doi: https://doi.org/10.14341/omet10331 